\title{
Circadian Rhythms in the CNS and Peripheral Clock Disorders: Function of Clock Genes: Influence of Medication for Bronchial Asthma on Circadian Gene
}

\author{
Naoto Burioka ${ }^{1, *}$, Yasushi Fukuoka ${ }^{1}$, Miyako Takata ${ }^{1}$, Masahiro Endo ${ }^{1}$, Masanori Miyata ${ }^{1}$, \\ Hiroki Chikumi ${ }^{1}$, Katsuyuki Tomita ${ }^{1}$, Masahiro Kodani ${ }^{1}$, Hirokazu Touge ${ }^{1}$, Kenichi Takeda ${ }^{1}$, \\ Takashi Sumikawa ${ }^{1}$, Kousuke Yamaguchi ${ }^{1}$, Yasuto Ueda ${ }^{1}$, Hirofumi Nakazaki ${ }^{1}$, Hisashi Suyama ${ }^{1}$, \\ Akira Yamasaki ${ }^{1}$, Hiroyuki Sano ${ }^{2}$, Tadashi Igishi ${ }^{1}$, and Eiji Shimizu ${ }^{1}$ \\ ${ }^{1}$ Division of Medical Oncology and Molecular Respirology, Faculty of Medicine, Tottori University, \\ 36-1 Nishimachi, Yonago 683-8504, Japan \\ ${ }^{2}$ Department of Respiratory Medicine Allergology, Kinki University School of Medicine, \\ Ohnohigashi 377-2, Sayama 589-8511, Japan
}

Received November 8, 2006; Accepted December 14, 2006

\begin{abstract}
Bronchial asthma is a chronic inflammatory disorder of the airways, in which inflammation causes bronchial hyper-responsiveness and flow limitation in the presence of various stimuli. Pulmonary function in asthmatic patients frequently deteriorates between midnight and early morning, which has suggested a role for chronotherapy. Although relationships between bronchial asthma and the function of clock genes remain unclear, some medications given for asthma such as glucocorticoids or $\beta_{2}$-adrenoceptor agonists may influence clock genes in vivo. In our studies of clock gene mRNA expressions in human bronchial epithelial cells in vitro and peripheral blood cells in vivo, we demonstrated that glucocorticoid or $\beta_{2}$-adrenoceptor agonist treatment strongly induced human Perl mRNA expression both in vitro and in vivo. Human peripheral blood cells provide a useful indication of peripheral clock gene mRNA expression in vivo.
\end{abstract}

Keywords: $\beta_{2}$-adrenoceptor agonist, cAMP, clock gene, glucocorticoid, Per 1

\section{Introduction}

Clock genes in the suprachiasmatic nucleus $(\mathrm{SCN})$ as well as in peripheral organs regulate important mammalian circadian rhythms $(1,2)$. Dysfunction of clock genes can contribute to sleep disorders, psychiatric diseases, tumor genesis, dyslipidemia, and cardiovascular disease $(2-5)$. Although molecular mechanisms involving the circadian oscillator in nocturnally active rodents have been studied in detail, few investigations have focused on diurnally active humans.

In bronchial asthma, chronic airway inflammation causes bronchial hyper-responsiveness and flow limitation in response to various external and internal stimuli.

*Corresponding author. burioka@grape.med.tottori-u.ac.jp Published online in J-STAGE: February 14, 2007

doi: 10.1254/jphs.FMJ06003X4
Since pulmonary function in asthma frequently is worst between midnight and the early morning $(6,7)$, chronotherapy has been used to alleviate this dysfunction. Glucocorticoids have been used clinically to control inflammation in asthma. Moreover, $\beta_{2}$-adrenergic receptors are widely distributed in the respiratory tract and airway smooth muscle (8), and $\beta_{2}$-adrenoceptor agonists have been used to improve symptoms and airway obstruction in bronchial asthma $(7,8)$.

Multiple signaling pathways can elicit expression of clock genes $(1,2)$, and several drugs can alter the molecular functions of these genes. In particular, Periodl (Perl) is an important circadian clock gene. Since Perl has both a glucocorticoid response element (GRE) $(9-11)$ and cAMP response element (CRE) in its 5 -upstream sequence $(12,13)$, administration of glucocorticoid or $\beta$-adrenoceptor agonist may induce Perl mRNA expression in vivo. Although asthma 
medications may influence clock genes, this has been unclear in humans. This minireview summarizes recent findings from our laboratory and others concerning influences of glucocorticoid and $\beta_{2}$-adrenoceptor agonist treatments on clock genes in humans.

\section{Molecular signals of the mammalian circadian clock gene Period1}

Circadian clock genes are expressed not only in the central pacemaker of the SCN but also in peripheral tissues such as kidney, liver, heart, skin, and peripheral blood mononuclear cells (PBMCs) $(14-18)$. In mammals, three clock genes, Per 1, Per2, and Per3, are expressed rhythmically in the SCN, and Perl and Per 2 are induced in response to light (19). Generally, negative feedback determines oscillation of circadian rhythm in both the SCN and peripheral tissues. BMAL1-CLOCK protein heterodimers are positive regulators that act through an E-box enhancer to activate transcription of Period, vasopressin, and $D B P$ mRNA. The proteins PER $1-3, \mathrm{CRY} 1-2, \mathrm{CKI} \varepsilon$, and DEC $1-2$ are negative regulators that inhibit activation of BMAL1-CLOCK heterodimers. $(20-22)$.

In addition, previous reports have shown that multiple signaling pathways, which elicit circadian expression of $P e r$ in cultured cells, are affected by retinoic acids, epidermal growth factor, glucocorticoids, equine serum in high concentration, forskolin, phorbol ester and sympathetic neuron-related factors $(1,23-28)$.

\section{Glucocorticoid induces human Period1 mRNA}

Glucocorticoids are a particularly potent signal eliciting rhythmic mRNA expression in peripheral clock genes. Rat Perl mRNA expression in cultured rat-1 fibroblasts was strongly induced by a glucocorticoid hormone analogue, dexamethasone $(1,23)$. This in-

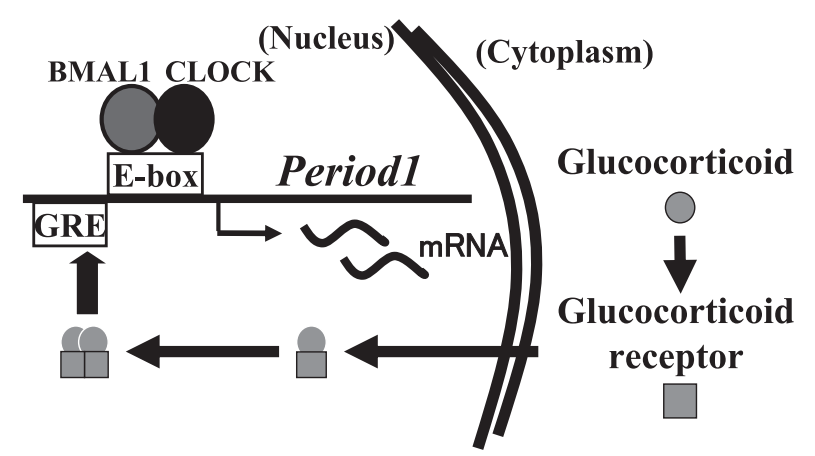

Fig. 1. Signaling pathway leading to Perl mRNA expression via the glucocorticoid response element (GRE). crease of Perl mRNA accumulation is caused by glucocorticoid signaling via GRE consensus sequences in Per1 $(9-11)$. Figure 1 shows the signaling pathway resulting in Perl mRNA expression via interaction with GRE.

Mechanisms of clock genes have been studied largely in nocturnally active rodents $(14,15,27,29)$. Much investigation remains to be carried out in diurnally active humans. Bjarnason et al. reported that clock genes such as hClock, hTim, hPerl, hCryl, and hBmall are expressed in human oral mucosa and skin (30). A rhythmic expression profile was found for human Perl, Cryl, and Bmall (30). However, biopsies to obtain samples of oral mucosa or skin from human subjects are limited by the need for local anesthesia when performing this procedure. Peripheral blood cells provide an easier way to evaluate changes in expression of peripheral clock genes. Recently, human PBMCs have been used as a surrogate to estimate likely mRNA expression of clock genes in other peripheral tissues $(16-18)$. The greatest $\mathrm{h}$ Per 1 transcription was observed in human subjects during the morning hours $(17,18,31)$.

We examined how stimulation with a glucocorticoid in vitro and in vivo affected expression of a clock gene, $\mathrm{h} P e r l$, in PBMCs. Exposure to dexamethasone strongly induced hPerl mRNA in cultured human bronchial epithelial cells, BEAS-2B; in contrast, hClock mRNA remained essentially unchanged after stimulation with dexamethasone (28). On average, relative $\mathrm{h}$ Per 1 mRNA expression according to the real-time polymerase chain reaction (PCR) was increased about 11 -fold by stimulation with dexamethasone (28). This result was consistent with the previous report (23). Furthermore, when human monocytes and lymphocytes were separated from PBMCs, and stimulated in vitro by prednisolone, both monocytes and lymphocytes showed induction of $\mathrm{hPerl}$ mRNA expression according to reverse-transcription (RT)-PCR (Fig. 2). Transcription of other clock genes, $\mathrm{h}$ Per2 and hClock, was not detectably induced by prednisolone (data not shown). Monocytes represent a relatively small fraction of PBMCs, so expression in monocytes and lymphocytes was assessed by the more sensitive real-time PCR method. Again, prednisolone at $10^{-7} \mathrm{M}$ induced expression of $\mathrm{hPerl}$ mRNA in both monocytes and lymphocytes (Fig. 3), but not hPer2 or hClock mRNA expression.

Figure 4 presents $\mathrm{hPer} 1 \mathrm{mRNA}$ expression in PBMCs from three healthy volunteers for 2 consecutive days (32). We first collected 10-ml venous blood samples at 9:00 and 10:00 AM and 12:00 and 9:00 PM. PBMCs were isolated immediately to determine baseline expression. On the second day, the volunteers were injected with $20 \mathrm{mg}$ of prednisolone at 9:00 AM, and separated 

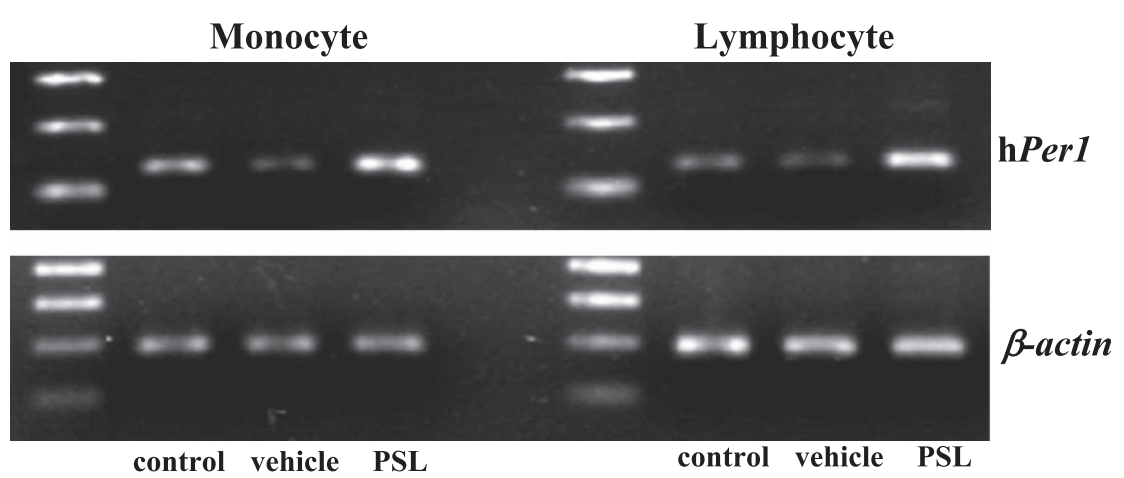

Fig. 2. RT-PCR analysis for mRNA expression of a human clock gene, hPerl. In both monocytes and lymphocytes, the hPerl mRNA expression was intensified by exposure in vitro to prednisolone (PSL, $10^{-7} \mathrm{M}$ ).
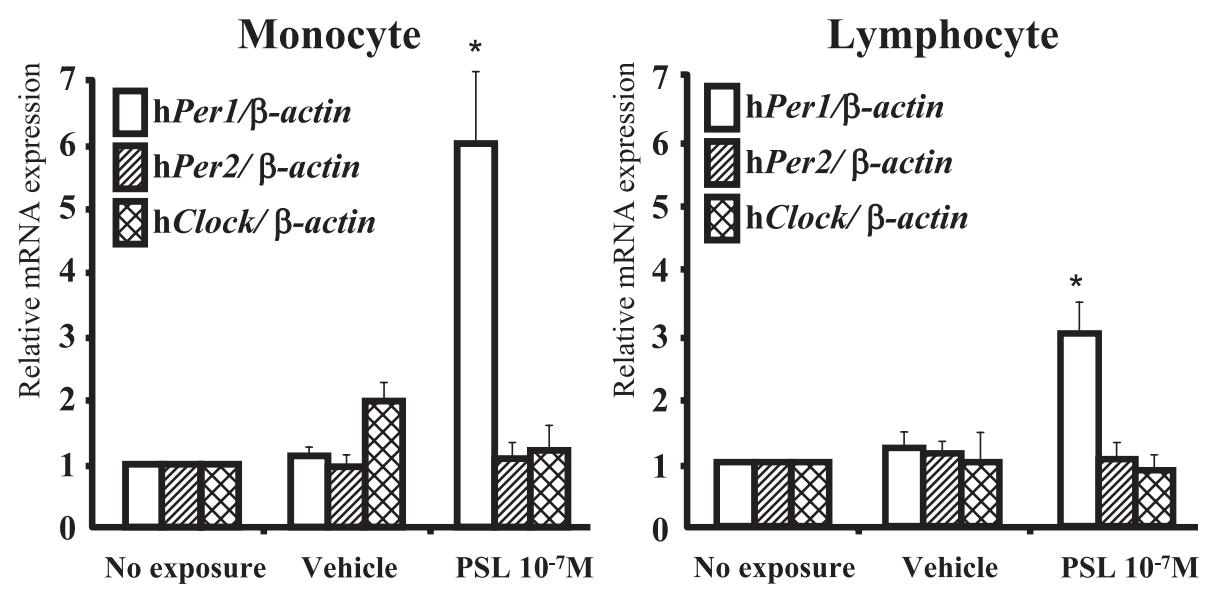

Fig. 3. Effect of prednisolone stimulation in vitro on human clock gene mRNA expression in monocytes and lymphocytes according to real-time PCR analysis. Monocyte and lymphocyte samples were exposed to vehicle alone (ethanol) or to $10^{-7} \mathrm{M}$ prednisolone (PSL) in vehicle for $1 \mathrm{~h}(\mathrm{n}=6$; mean \pm S.E.M.). Lymphocytes showed an increase similar to that in monocytes, but only about half as intense. ${ }^{*} P<0.04$, compared to the relative values with vehicle alone (Wilcoxon test).

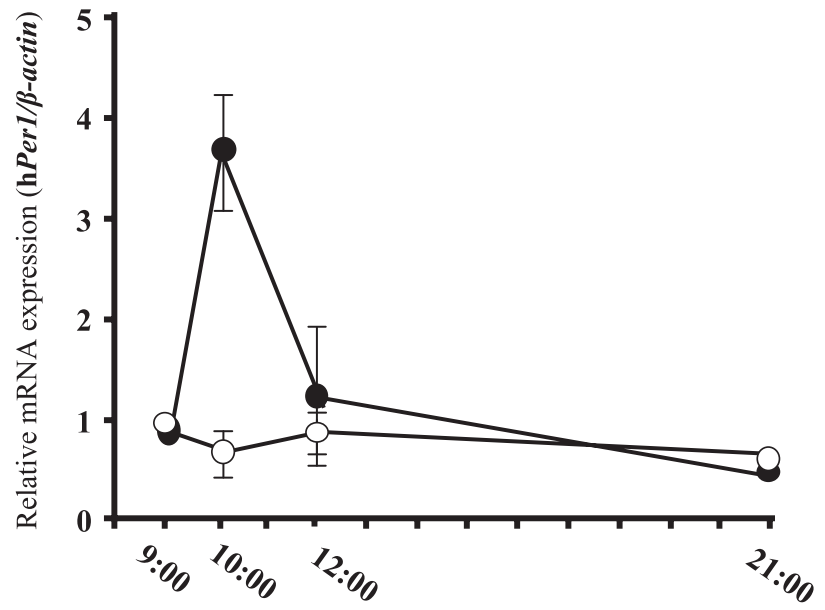

Fig. 4. Induction of hPerl mRNA by prednisolone injection in three healthy subjects according to real-time PCR analysis. Expression of $\mathrm{hPer} 1$ mRNA in PBMCs was assessed at four time points on each of 2 days in three healthy subjects (mean \pm S.D.). Expression at 9:00 AM on day 1 was defined as 1 (baseline). Open symbols indicate day 1 (no prednisolone), while closed symbols indicate day 2 (prednisolone given at 9:00 AM).
PBMCs were obtained at the same four times. We found that $\mathrm{h}$ Per 1 mRNA expression in PBMCs was strongly induced by prednisolone in vivo, while $\mathrm{hPer} 2$ and hClock mRNA expressions were not induced (32). Expression of hPerl mRNA in PBMCs then rapidly decreased, returning to baseline by $12 \mathrm{~h}$. Thus, the influence of glucocorticoids on clock genes was confirmed in PBMCs exposed to them in vivo. In a previous study of nocturnally active rodents, administration of prednisolone at ZT0 for 7 days altered hepatic mRNA expression rhythms of $\mathrm{mPer} 1, \mathrm{mPer} 2, \mathrm{mRev-erb \alpha}$, and $\mathrm{m}$ Bmall, but administration of ZT12 had no significant effect on expression rhythms of clock genes (11). Since secretion of endogenous glucocorticoid hormones in rodent peaks from the late light phase to the early dark phase, administration of prednisolone at ZT0 would be likely to disturb the endogenous glucocorticoid rhythm and thus alter expression of clock genes in the liver (11). In diurnally active humans, the secretion of endogenous glucocorticoid hormones peaks early in the morning, so morning administration of glucocorticoid would be less 
likely to influence clock genes. Although responses to glucocorticoid stimulation may differ between organs, human PBMCs are readily available to examine changes in mRNA transcription from clock genes.

\section{$\beta_{2}$-Adrenoceptor agonist induces human Period1 mRNA}

Factors related to sympathetic neurons recently have been reported to reset the clock genes (26). Many sympathetic nervous system functions are mediated by adrenoceptor signaling. Expressions of the mouse Perl gene were induced by an $\alpha$-adrenoceptor agonist or a $\beta$ adrenoceptor agonist, but attenuated by a mitogenactivated protein kinase (MAPK) kinase inhibitor or protein kinase A (PKA) inhibitor (26). Induction of Perl gene expression in cultured cells is related to cAMP response element binding protein (CREB) phosphorylation via activation of MAPK and PKA $(12,24,26,33)$. Yagita and Okamura (24) reported that forskolin induced phosphorylation of CREB in rat-1 cells. Forskolin directly activates adenylate cyclase, which enhances the synthesis of native cAMP and the indirect activation of PKA. This pathway activates CREB by phosphorylation. Moreover, they revealed that forskolin induced $\mathrm{r}$ Per 1 but not $\mathrm{r}$ Per 2 mRNA in rat- 1 cells.

The human $\beta$-adrenoceptor belongs to a family of receptors with seven-transmembrane domains and is classified into $\beta_{1}, \beta_{2}$, and $\beta_{3}$ subgroups. In particular, $\beta_{2-}$ adrenoceptors are widely distributed in the respiratory tract and airway smooth muscle (8). Intracellular signaling following $\beta_{2}$-adrenoceptor activation is influenced by Gs protein coupled to adenylate cyclase. Airway relaxation is induced by cAMP through phosphorylation of muscle regulatory proteins and CREB via PKA and through reduction of cellular $\mathrm{Ca}^{2+}$ concentrations (8). Agonists of $\beta_{2}$-adrenoceptors have been used to reduce airway obstruction in patients with bronchial asthma (7, $8)$. However, the influence of $\beta_{2}$-adrenoceptor agonists on clock genes has been unclear.

We have demonstrated that $\beta_{2}$-adrenoceptor agonists strongly induced $\mathrm{h}$ Per $1 \mathrm{mRNA}$ expression in the human bronchial epithelial cell line BEAS-2B, using real-time PCR analysis and detected phosphorylated CREB by immunoblotting (13). In brief, to detect phosphorylation of CREB in BEAS-2B cells by fenoterol or procaterol, immunoblotting was performed using an anti-phosphoCREB-specific antibody after exposure of the cells to $\beta_{2^{-}}$ adrenoceptor agonists. Fenoterol or procaterol induced phosphorylation of CREB, although the total amount of CREB was unchanged (13). Figure 5 demonstrates induction of $\mathrm{h}$ Perl mRNA by fenoterol or procaterol $\left(10^{-7} \mathrm{M}\right)$ in BEAS-2B cells according to real-time PCR

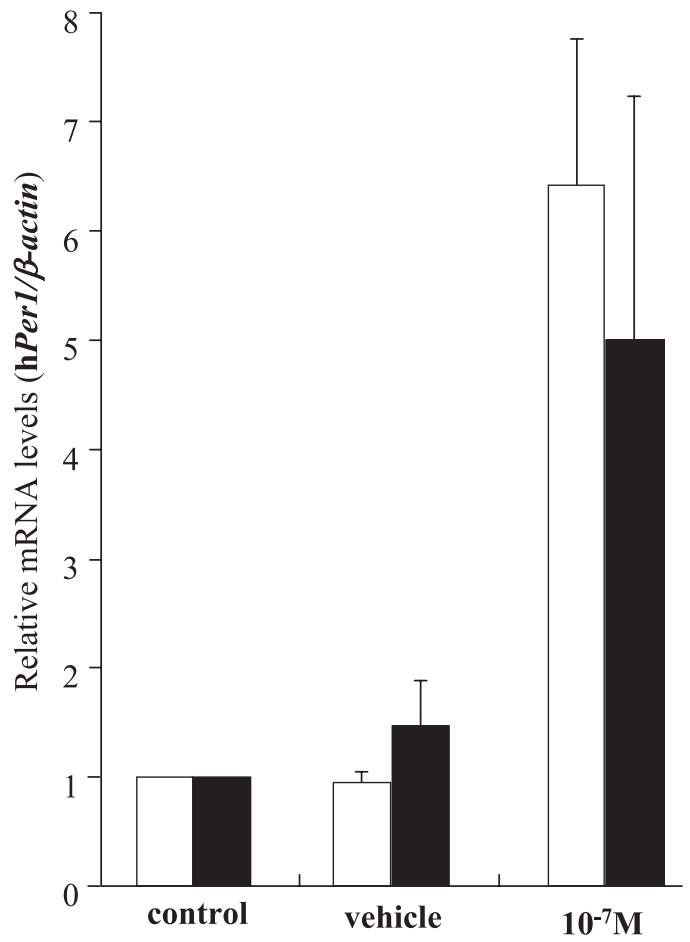

Fig. 5. Effect of fenoterol or procaterol on the $\mathrm{h}$ Perl mRNA induction in BEAS-2B cells according to real-time PCR. Cells that had been pre-incubated with serum-free DMEM for $24 \mathrm{~h}$ were stimulated with fenoterol or procaterol for $1 \mathrm{~h}$. Expression of $\mathrm{h} P$ er 1 mRNA was normalized to expression of $\beta$-actin mRNA in real-time PCR analysis. White and black bars indicate the results of fenoterol and procaterol treatment, respectively. Data are shown as means \pm S.D. $(n=4$ for fenoterol, $n=3$ for procaterol).

analysis, with $\mathrm{hPer} 1$ expression normalized to expression of $\beta$-actin. This demonstrated that $\beta_{2}$-adrenoceptor agonists strongly induced $\mathrm{hPer} 1 \mathrm{mRNA}$ expression in BEAS-2B cells via the cAMP-CREB signaling pathway (13). A previous report suggested that the changes in noradrenaline, an $\alpha$ - and $\beta$-adrenoceptor agonist, maintained $\mathrm{mPer} 1$ and $\mathrm{mPer} 2$ oscillation in mouse liver over the course of a day (26). In humans, the influence of $\beta_{2-}$ adrenoceptor agonists on clock genes in lungs is still unclear, requiring further study. Figure 6 shows the signaling pathway leading to expression of Perl via its CRE.

We also examined induction of human clock genes in vivo by exogenous $\beta_{2}$-adrenoceptor agonists in healthy subjects. When a $\beta_{2}$-adrenoceptor agonist is administered at night, Per $1 \mathrm{mRNA}$ expression could be greatly influenced (data not shown). Recently, a transdermal system for once-daily evening dosing of tulobuterol was developed in Japan (Hokunarin tape ${ }^{\circledR}$; Abott Japan Pharmaceutical, Tokyo). The tulobuterol patch attached to the skin in early evening then achieves a peak blood concentration after 9 to $12 \mathrm{~h}$ (34). This slow-release drug 


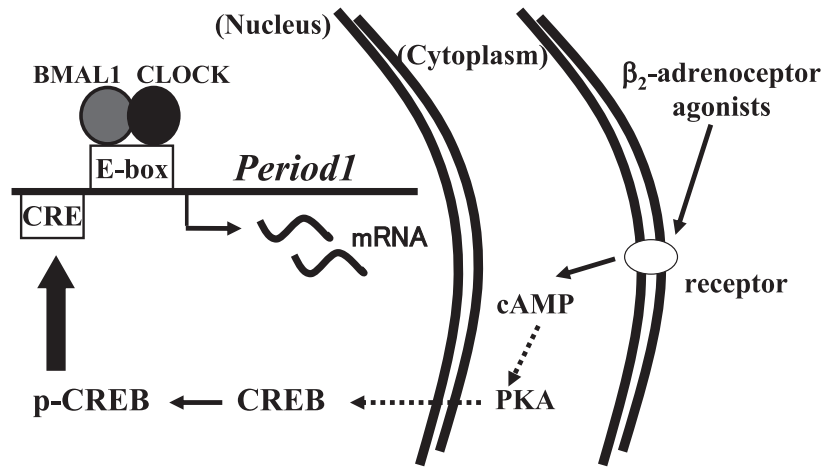

Fig. 6. Signaling pathway leading to Perl mRNA expression via the cAMP response element (CRE).

delivery system is designed for use as chronotherapy for nocturnal asthma (7). We found that the influence of tulobuterol transdermal patch on $\mathrm{h} P$ er 1 mRNA expression in whole peripheral blood cells sampled at night was less than that of oral tulobuterol tablet (data not shown).

\section{Conclusions}

In summary, human peripheral blood cells may be used as a surrogate for clock gene mRNA expression in other peripheral tissues. Moreover, monitoring of clock genes rhythmicity in peripheral blood cells may be useful for choosing the most appropriate time of day for giving certain medications to patients with bronchial asthma, aiming to increase therapeutic effects and/or reduce adverse effects. Specialized drug delivery systems may attenuate excessive influence of some drugs on clock genes. However, the actual physiologic impacts on clock genes by medications given to patients with asthma remain a matter for future study.

\section{Acknowledgment}

This study was partly supported by a grant (17590791) from the Ministry of Education, Culture, Sports, Science, and Technology of Japan (to N.B.).

\section{References}

1 Balsalobre A. Clock genes in mammalian peripheral tissues. Cell Tissues Res. 2002;309:193-199.

2 Cermakian N, Boivin DB. A molecular perspective of human circadian rhythm disorders. Brain Res Brain Res Rev. 2003; 42:204-220.

$3 \mathrm{Fu} \mathrm{L}$, Lee CC. The circadian clock: pacemaker and tumour suppressor. Nat Rev Cancer. 2003;3:350-361.

4 Shimba S, Ishii N, Ohta Y, Ohno T, Watabe Y, Hayashi M, et al. Brain and muscle Arnt-like protein-1 (BMAL1), a component of the molecular clock, regulates adipogenesis. Proc Natl Acad Sci U S A. 2005;102:12071-12076.

5 Young ME. The circadian clock within the heart: potential influence on myocardial gene expression, metabolism, and function. Am J Physiol Heart Circ Physiol. 2006;290:H1-H16.

6 Burioka N, Suyama H, Sako T, Shimizu E. Circadian rhythm in peak expiratory flow: Alteration with nocturnal asthma and theophylline chronotherapy. Chronobiol Int. 2000;17:513-519.

7 Burioka N, Miyata M, Endo M, Fukuoka Y, Suyama H, Nakazaki H, et al. Alteration of the circadian rhythms in peak expiratory flow of nocturnal asthma following nighttime transdermal $\beta_{2}$-adrenoceptor agonist tulobuterol chronotherapy. Chronobiol Int. 2005;22:353-360.

8 Johnson M. The $\beta$-adrenoceptor. Am J Respir Crit Care Med. 1998;158:s146-s153.

9 Le Minh N, Damiola F, Tronche F, Schutz G, Schibler U Glucocorticoid hormones inhibit food-induced phase-shifting of peripheral circadian oscillators. EMBO J. 2001;20:7128-7136.

10 Hida A, Koike N, Hirose M, Hattori M, Sakaki Y, Tei H. The human and mouse Period 1 genes: five well-conserved E-boxes additively contribute to the enhancement of mPer1 transcription. Genomics. 2000;65:224-233.

11 Koyanagi S, Okazawa S, Kuramoto Y, Ushijima K, Shimeno H, Soeda $\mathrm{S}$, et al. Chronic treatment with prednisolone represses the circadian oscillation of clock gene expression in mouse peripheral tissues. Mol Endocrinol. 2006;20:573-583.

12 Travnickova-Bendova Z, Cermakian N, Reppert SM, SassoneCorsi P. Bimodal regulation of mPeriod promoters by CREBdependent signaling and CLOCK/BMAL1 activity. Proc Natl Acad Sci U S A. 2002;99:7728-7733.

13 Takata M, Burioka N, Ohdo S, Fukuoka Y, Miyata M, Endo M, et al. $\beta_{2}$-adrenoceptor agonists induce the mammalian clock gene, hPerl, mRNA in cultured human bronchial epithelium cells in vitro. Chronobiol Int. 2005;22:777-783.

14 Shibata S. Neural regulation of the hepatic circadian rhythm. Anat Rec A Discov Mol Cell Evol Biol. 2004;280:901-909.

15 Oishi K, Sakamoto K, Okada T, Nagase T, Ishida N. Humoral signals mediate the circadian expression of rat period homologue (rPer2) mRNA in peripheral tissues. Neurosci Lett. 1998;256: $117-119$.

16 Takata M, Burioka N, Ohdo S, Takane H, Terazono H, Miyata $\mathrm{M}$, et al. Daily expression of mRNAs for the mammalian clock genes Per2 and clock in mouse suprachiasmatic nuclei and liver and human peripheral blood mononuclear cells. Jpn J Pharmacol. 2002;90:263-269.

17 Boivin DB, James FO, Wu A, Cho-Park PF, Xiong H, Sun ZS. Circadian clock genes oscillate in human peripheral blood mononuclear cells. Blood. 2003;102:4143-4145.

18 Kusanagi H, Mishima K, Satoh K, Echizenya M, Katoh T, Shimizu T. Similar profiles in human period 1 gene expression in peripheral mononuclear and polymorphonuclear cells. Neurosci Lett. 2004;22;365:124-127.

19 Zylka MJ, Shearman LP, Weaver DR, Reppert SM. Three period homologs in mammals: differential light responses in the suprachiasmatic circadian clock and oscillating transcripts outside of brain. Neuron. 1998;20:1103-1110

20 Dunlap JC. Molecular bases for circadian clocks. Cell. 1999;96:271-290.

21 Shearman LP, Sriram S, Weaver DR, Maywood ES, Chaves I, Zheng $\mathrm{B}$, et al. Interacting molecular loops in the mammalian 
circadian clock. Science. 2000;288:1013-1019.

22 Yagita K, Yamaguchi S, Tamanini F, van der Horst GTJ, Hoeijmakers JHJ, Yasui A, et al. Dimerization and nuclear entry of mPER proteins in mammalian cells. Genes Dev. 2000;14:1353-1363.

23 Balsalobre A, Brown SA, Marcacci L, Tronche F, Kellendonk C, Reichardt HM, et al. Resetting of circadian time in peripheral tissues by glucocorticoid signaling. Science. 2000;289:23442347.

24 Yagita K, Okamura H. Forskolin induces circadian gene expression of rPer1, rPer2 and dbp in mammalian rat-1 fibroblasts. FEBS Lett. 2000;465:79-82.

25 Oh-hashi K, Naruse Y, Tanaka M. Intracellular calcium mobilization induces period genes via MAP kinase pathways in NIH3T3 cells. FEBS Lett. 2002;516:101-105.

26 Terazono H, Mutoh T, Yamaguchi S, Kobayashi M, Akiyama M, Udo R, et al. Adrenergic regulation of clock gene expression in mouse liver. Proc Natl Acad Sci U S A. 2003;100:6795-6800.

27 Karolczak M, Burbach GJ, Sties G, Korf HW, Stehle JH. Clock gene mRNA and protein rhythms in the pineal gland of mice. Eur J Neurosci. 2004;19:3382-3388.

28 Burioka N, Takata M, Okano Y, Ohdo S, Fukuoka Y, Miyata M, et al. Dexamethasone influences human clock gene expression in bronchial epithelium and peripheral blood mononuclear cells in vitro. Chronobiol Int. 2005;22:585-590.

29 Ohdo S, Koyanagi S, Suyama H, Higuchi S, Aramaki H. Changing the dosing schedule minimizes the disruptive effects of interferon on clock function. Nature Med. 2001;7:356-360.

30 Bjarnason GA, Jordan RCK, Wood PA, Li Q, Lincoln DW, Sothern RB, et al. Circadian expression of clock genes in human oral mucosa and skin. Am J Pathol. 2001;158:1793-1801.

31 Takimoto M, Hamada A, Tomoda A, Ohdo S, Ohmura T, Sakato $\mathrm{H}$, et al. Daily expression of clock genes in whole blood cells in healthy subjects and a patient with circadian rhythm sleep disorder. Am J Physiol Regul Integr Comp Physiol. 2005; 289:R1273-R1279.

32 Fukuoka Y, Burioka N, Takata M, Ohdo S, Miyata M, Endo M, et al. Glucocorticoid increases hPer1 mRNA levels in human peripheral blood mononuclear cells in vitro or in vivo. $\mathrm{J}$ Biol Rhythm. 2005;20:550-553.

33 Coogan AN, Piggins HD. MAP kinases in the mammalian circadian system-key regulators of clock function. J Neurochem. 2004;90:769-775.

34 Uematsu T, Nakano M, Kosuge M, Kanamura M, Nakashima M. The pharmacokinetics of $\beta_{2}$-adrenoceptor agonist, tulobuterol, given transdermally and by inhalation. Eur J Clin Pharmacol. 1993;44:361-364. 